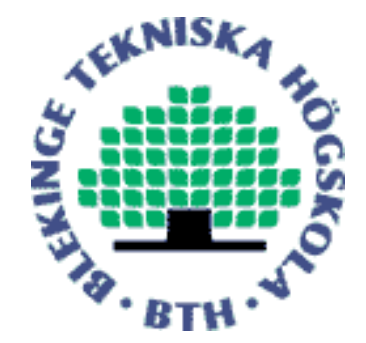

Copyright (C) 2014 IEEE.

Citation for the published paper:

Cooperative multiple-access scheme with antenna selection and incremental relaying

Dimas I. Alves, Renato Machado, Andrei P. Legg, Bartolomeu F. Uchoa-Filho

Telecommunications Symposium (ITS), 2014 International

2014 São Paulo

This material is posted here with permission of the IEEE. Such permission of the IEEE does not in any way imply IEEE endorsement of any of BTH's products or services Internal or personal use of this material is permitted. However, permission to reprint/republish this material for advertising or promotional purposes or for creating new collective works for resale or redistribution must be obtained from the IEEE by sending a blank email message to pubs-permissions@iee.org.

By choosing to view this document, you agree to all provisions of the copyright laws protecting it. 


\title{
Cooperative Multiple-Access Scheme with Antenna Selection and Incremental Relaying
}

\author{
Dimas Irion Alves*, Renato Machado ${ }^{\dagger \ddagger}$, Andrei Piccinini Legg ${ }^{\ddagger}$, and Bartolomeu F. Uchôa-Filho* \\ * Federal University of Santa Catarina - UFSC \\ Florianøpolis, SC, 88040-900, Brazil \\ $\dagger$ Blekinge Institute of Technology - BTH \\ Karlskrona, SE-371 79, Sweden. \\ $\ddagger$ Federal University of Santa Maria - UFSM \\ Santa Maria, RS, 97105-900, Brazil. \\ E-mail: dimasirion977@gmail.com, renatomachado@ieee.org, andrei.legg@gmail.com, uchoa@iee.org
}

\begin{abstract}
A cooperative multiple-access scheme for wireless communications systems with antenna selection and incremental relaying is proposed. The scheme aims to improve the system throughput while preserving good performance in terms of bit error rate. The system consists of $N$ nodes which send their information to both the destination node and the multipleantenna relay station. Based on the channel state information, the destination node decides whether or not relaying will be performed. When the relaying is performed, the decoderemodulate-and-forward protocol is used with the best antenna. Results reveal that the proposed scheme achieves a good tradeoff between throughput and bit error rate, which makes suitable to be considered for multi-user networks.
\end{abstract}

\section{INTRODUCTION}

The wireless communications technology has reached a level of complexity where the traditional point-to-point communication systems are not able to respond the increasing demand imposed by the market anymore. Owing to this issue, there has been a significant increase of interest in developing new architectures and techniques by exploring the broadcast nature of the wireless medium [1], [2], where new strategies can be considered to explore the spatial diversity provided by relay nodes which are usually distributed between source and destination [3], [4], [5].

Although the use of cooperative schemes can reduce the negative effect of fading, it can also decrease the system spectral efficiency. To remedy this problem, interesting solutions for multiple-access channel (MAC) based on joint transmission [6], relay selection [7] and node selection [8] have been proposed. These ones can be used to expand the system coverage area, extend the battery life time, increase the system reliability, and increase the system transmission rate. Recently, several segments with a great variety of applications, such as vehicular communications, cellular networks, sensor networks, and satellite communications, are taking into consideration the use of those techniques in order to improve the system performance [5], [9].

Due to the large range of applications of wireless communication systems, several works have been proposed new transmit schemes for multi-node and feedback-assisted relaying systems. Some of them are briefly described in the sequel.

\section{A. Related Works}

In [10], an incremental relaying protocol based on the relay selection technique was proposed where, for each new frame and based on the instantaneous channel conditions, the destination node informs both the relays and the source nodes whether or not relaying is to be performed, and if so, which relay must act (namely, the relay with the best relay-to-destination channel quality). The technique presented in [10] provides a good tradeoff between spectral efficiency and cooperative diversity and it can be used along with both Amplify-and-Forward (AF) and Decode-and-Forward (DF) protocols.

In [11], the selective decode-and-forward (SDF) protocol is presented. In this scheme, the source nodes broadcast their information to the relay and the destination node in the first time slot. In the second time slot, if the channel quality is above a certain threshold, the relay retransmits a decoded version of the source signal to the destination node, otherwise the relay remains in silence.

The decode-remodulate-and-forward (DreMF) protocol proposed in [12] is similar to the DF protocol, however, in the DF protocol the relay decode the signal before forwarding it, while in the DreMF the relay performs a remodulation of the source signal into a different constellation. Note that the DreMF can be used to improve the spatial diversity order of the system as well as to increase the spectral efficiency if the decoded signal is remodulated into a high order constellation.

In [13] a design method is proposed for cooperative networks with multiple sources and multiple relays, where a different diversity order can be associated to each source node, depending on what quality-of-service $(\mathrm{QoS})$ it desires/requires; the analytical performance is presented in [14].

Considering a multiple-access DF relay system, in [15] a superposition code design is proposed, where an hierarchical modulation is used in order to reduce the degradation caused by the power reduction per transmit node. In [16], a space-time block code scheme for a multiple-access network is presented which provides a good tradeoff between decoding complexity and information loss. In [17], a network coding is proposed for a multiple-access relay network based on the information bottleneck method [18], which is considered in this work. 


\section{B. Contributions and Objectives}

We propose a multiuser multiple-access incremental relaying scheme based on the DreMF protocol and the antenna selection technique. The proposed scheme aims to provide a good solution for networks with a large number of sources, keeping a good balance among transmission rate, user batteries life-time and system reliability. A good example of such application is a vehicular communications network, which is generally composed of a lot of sources (vehicles) with extended battery life-time and abundant computational resources; the requirements of those networks are transmission rate and information reliability. Another example is the sensor networks which have a large number of nodes that can be described as users. This kind of system has limited computational resources and the users battery consumption is a major concern.

Based on the practical applicability of the proposed scheme, its performance is evaluated and some tradeoff issues related to transmission rate, bit error rate and transmission power are discussed.

\section{Organization}

The remainder of this paper is organized as follows. Section II introduces the system model considered in this work. Section III presents the cooperative multiple-access channel scheme with antenna selection. In Section IV, simulation results are presented and discussions are provided. Finally, Section V presents some concluding remarks.

\section{System Model Description}

The system model considered in this work is presented in Fig.1. The system is composed of $N$ source nodes, $S_{i}$, where $i=1, \ldots, N$, one multiple-antenna relay station, $R$, with $M$ antennas and one destination node, $D$, equipped with a single antenna.

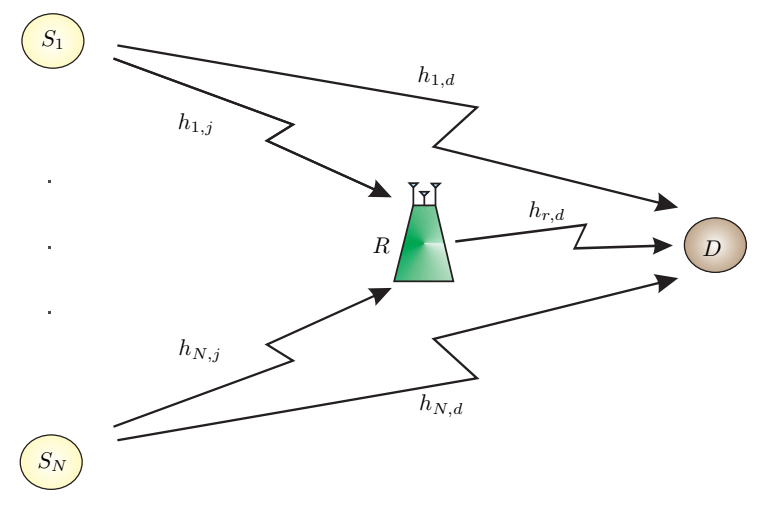

Fig. 1. System model with a multiple-antenna relay station.

The channels are considered to undergo quasi-static, flat Rayleigh fading so that the channels do not change over a frame and change independently and randomly from one frame to another. It is also assumed that the information bits are mapped into a baseband signal constellation with unity average energy, such as phase shift keying (PSK) or quadrature amplitude modulation (QAM).
We also assume that $i$ ) the channel fading coefficients are known at the receivers, $i i$ ) the nodes operate in half-duplex mode, $i$ ii) the transmission mode is the time-division multipleaccess relay channel (MARC) protocol [17], where source nodes and relay node transmit at different time-slots, $i v$ ) the total transmit power per transmission period is $P, v$ ) an errorfree feedback channel between the destination and the relay is available, and $v i$ ) the transmissions are synchronized.

\section{PROposed SCHEME}

The transmission is performed as follows. In the first timeslot, all the $N$ source nodes simultaneously broadcast their information to both the relay node and the destination node. The information of source node $i$ consists of a vector (frame) of $L$ symbols taken from a signal constellation $S$. The vectors received at the $j$-th antenna of the relay station and at the destination node, respectively, can be written as

$$
\mathbf{y}_{s, r, j}=\sum_{i=1}^{N} \sqrt{P_{1}} h_{i, j} \mathbf{s}_{i}+\eta_{s, r}
$$

and

$$
\mathbf{y}_{s, d}=\sum_{i=1}^{N} \sqrt{P_{1}} h_{i, d} \mathbf{s}_{i}+\eta_{s, d}
$$

where $\mathbf{s}_{i}$ is the signal vector transmitted by the $i$-th source node, $h_{i, j}$ and $h_{i, d}$ denote the channel coefficients from the $i$-th source to the $j$-th antenna at the relay station and to the destination node, respectively. $\eta_{s, r}$ and $\eta_{s, d}$ are the independent and identically distributed (i.i.d) zero-mean complex Gaussian noise vector with covariance matrix $N_{0} \mathbf{I}_{L}$, and $P_{1}$ represents the transmit power at the transmit nodes.

In this paper we assume that the transmit power is uniformly distributed among the $N$ source nodes. Thus, $P_{1}=$ $P / N$. Further, we assume that all source symbols belong to a same constellation. However, this is a constraint that can be removed.

Throughout this paper, for simplicity of exposition, uncoded transmission is assumed. In this case, the maximum likelihood (ML) detections at the relay station and at the destination node are, respectively, given as

$$
\left\{\hat{\mathbf{s}}_{r, i}\right\}_{i=1}^{N}=\arg \min _{\substack{\mathbf{s}_{i}^{\prime} \in S^{L} \\ i=1, \ldots, N}}\left[\sum_{j=1}^{M}\left|\mathbf{y}_{s, r, j}-\sum_{i=1}^{N}\left(\sqrt{P_{1}} h_{i, j} \mathbf{s}_{i}^{\prime}\right)\right|^{2}\right]
$$

and

$$
\left\{\hat{\mathbf{s}}_{d, i}\right\}_{i=1}^{N}=\arg \min _{\substack{\mathbf{s}_{i}^{\prime} \in S^{L} \\ i=1, \ldots, N}}\left[\left|\mathbf{y}_{s, d}-\sum_{i=1}^{N}\left(\sqrt{P_{1}} h_{i, d} \mathbf{s}_{i}^{\prime}\right)\right|^{2}\right] .
$$

In practice, however, each vector $\mathbf{s}_{i}$ is in fact a channel encoded packet whose redundancy can be used to correct a few errors at the symbol level as well as to check the packet integrity (say, for instance, via cyclic-redundant check (CRC) codes) at the destination. In this case, the ML detections in (3) and (4) must be replaced by the corresponding decoders.

In the proposed scheme, the destination node checks whether or not the $N$ frames have been recovered without 
errors. Then, as part of the proposed protocol, the destination node sends a message through a quantized feedback channel informing all other nodes in the network whether or not there was an error. If an error has occurred, then the destination node also informs which antenna of the relay node forms with the destination node the channel with the highest instantaneous signal-to-noise ratio (SNR). The number of bits sent through the feedback channel is then given by

$$
b=\left\lceil\log _{2}(M+1)\right\rceil,
$$

where $M$ is the number of antennas at the relay node. In the second time slot frame, if there is one, the relay station performs the DreMF protocol. For simplicity's sake, assume that all the source symbols are mapped into the BPSK constellation. Thus, the relay station will combine and remodulate these symbols into a higher order constellation $S_{h}$. For three sources or less, the symbols are re-mapped to a higher order PSK constellation, and for more than three sources, to a higher order QAM constellation. For example, if we have two or three source nodes, the higher order constellations are QPSK and 8-PSK, respectively. If we are assuming four source nodes, then the symbols are re-mapped into a 16-QAM constellation. Hence, the symbols transmitted by the sources are re-mapped always into a single symbol. Of course, the relay node may be required to send more than one symbol if the number of source nodes is large. But we shall not consider this case herein.

The vector received by the destination node in the second time-slot can be written as

$$
\mathbf{y}_{r, d}=\sqrt{P_{2}} h_{r, d} \hat{\mathbf{s}}_{r}+\eta_{r, d}
$$

where $\hat{\mathbf{s}}_{r} \in S_{h}^{L}$ is the remodulated vector transmitted by the relay station to the destination node, $h_{r, d}$ denotes the best channel coefficient between a relay station antenna and the destination node, $\eta_{r, d}$ is the independent and identically distributed (i.i.d) zero-mean complex Gaussian vector noise with covariance matrix $N_{0} \mathbf{I}_{L}$, and $P_{2}$ represents the transmit power of the relay station.

The ML detection for the second time slot is given as

$$
\hat{\mathbf{r}}_{d}=\arg \min _{\hat{\mathbf{s}}_{k} \in S_{h}^{L}}\left|\mathbf{y}_{r, d}-\left(\sqrt{P_{2}} h_{r, d} \hat{\mathbf{s}}_{k}\right)\right|^{2},
$$

where $S_{h}$ is the higher order constellation, which depends on the constellations that have been used by the source nodes.

It should be noted that it is very likely that just a small number of packets are declared as erroneous by the destination node. This means that the destination node needs much less redundant information than what it gets in the second time slot, which is a waste of resources. Therefore, a refinement of the proposed scheme may be obtained if the destination node sends more feedback bits to inform the relay station which information must be sent and which information can be discarded, optimizing even more the system. However, we shall not consider this case herein. Besides, we are assuming that the feedback channel is errorless, i.e., only a few number of feedback bits can be sent though the feedback channel.

\section{Simulation Results}

This section presents some simulation results in order to assess the proposed scheme performance. For simplicity, we assume that the frames consists of a single symbol, i.e., $L=1$. We run Monte Carlo simulations with the transmission of $10^{7}$ symbols by each source node per average SNR. It is assumed that the source symbols are mapped into a BPSK constellation and the transmitted power is $P_{1}=P / N$ and $P_{2}=P$. It is also assumed a perfect error detection scheme at the destination node. The transmission rate is defined here as $R=b / T$, where $b$ is the number of transmitted bits and $T$ is the number of time slots. It is important to say that $T$ depends on the protocol that is used to transmit the information.

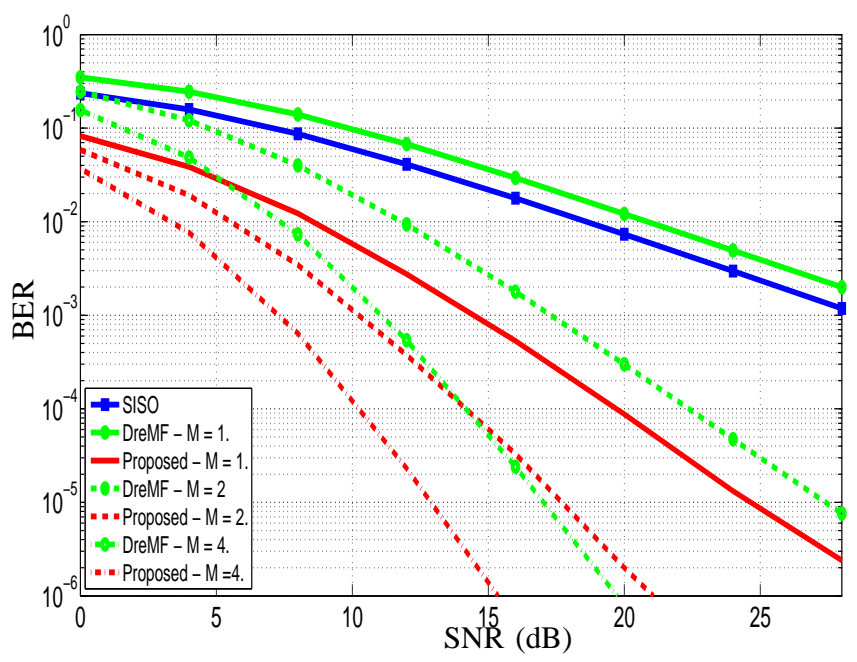

Fig. 2. BER performance of the proposed scheme. $\mathrm{N}=2$ source nodes.

The bit error rate (BER) performance for the proposed scheme, with $N=2,3$ and 4 and for $M=1,2$ and 4 , is presented in Figs. 2-4. The results illustrate that the proposed scheme outperforms the SISO and the DreMF schemes. Also, one can see that the proposed scheme has an improved diversity when compared with the DreMF scheme.

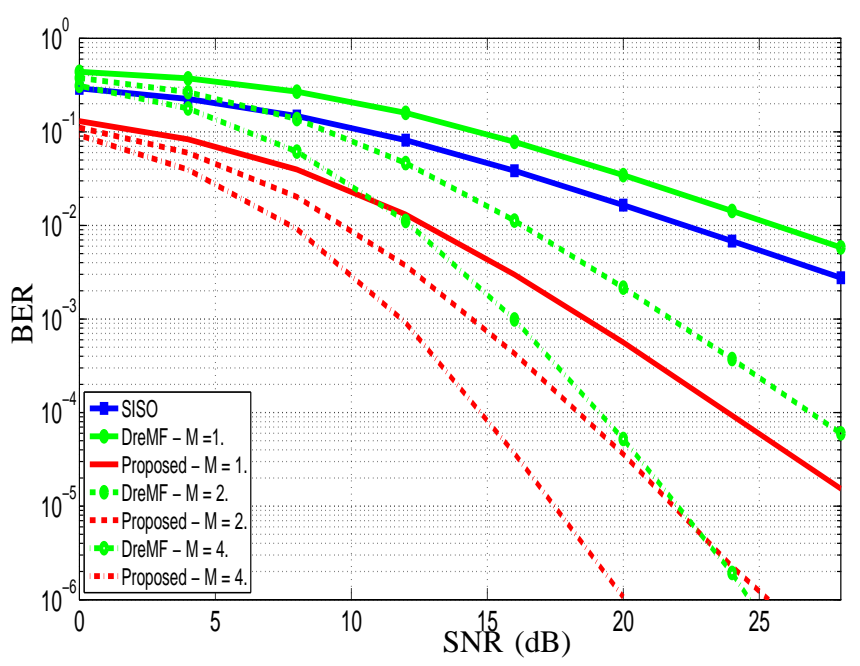

Fig. 3. BER performance of the proposed scheme. $\mathrm{N}=3$ source nodes. 


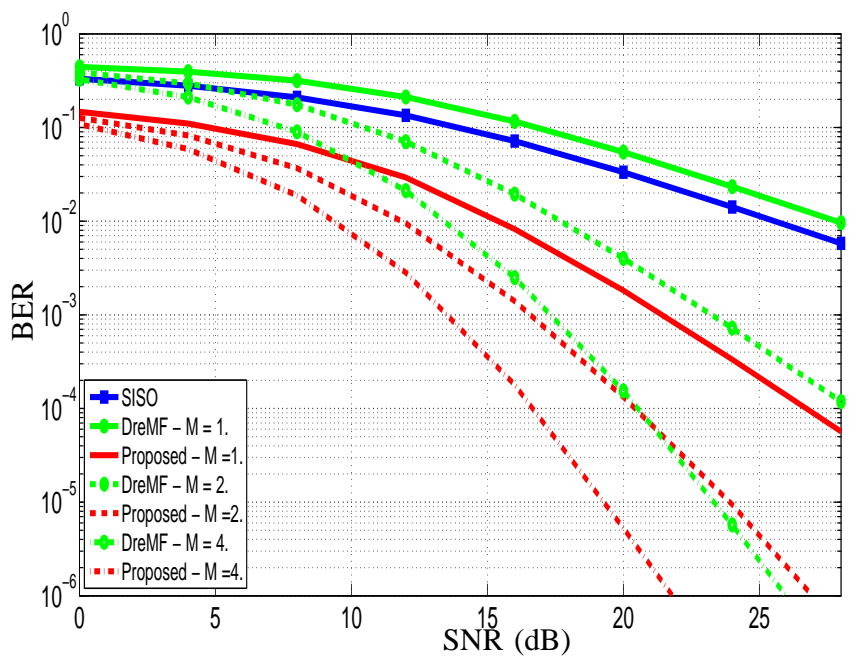

Fig. 4. BER performance of the proposed scheme. $\mathrm{N}=4$ source nodes.

TABLE I presents the average SNR of the proposed scheme for a BER of $10^{-5}$ and $M=4$ transmit antennas at the relay station. It is noticeable that the average SNR required of the proposed scheme scheme increases as the number of sources is augmented. From this result, we can say that for maintaining a good BER performance when the number of source nodes is large, one can increase the number of antennas allocated at the relay station. Another option is to consider transmit power allocation in order to optimize the instantaneous SNR at the destination.

TABLE I. AVERAGE SNR FOR A BER OF $10^{-5}$ AND $M=4$.

\begin{tabular}{c|c}
\hline Number of Sources & Average SNR \\
\hline$N=2$ & $12.89 \mathrm{~dB}$ \\
\hline$N=3$ & $17.47 \mathrm{~dB}$ \\
\hline$N=4$ & $19.27 \mathrm{~dB}$ \\
\hline
\end{tabular}

Fig. 5 and TABLE II presents, respectively, the transmission rate and the relay usage for the same system configuration as considered in Figs. 2-4. According to TABLE III, one can see that as the number of transmit sources increases, the transmission rate decreases. This occurs due to the transmission power constraint, where the total transmit power is divided by the number of source nodes. Wherefore, as mentioned before, if a higher transmission rate is required for a large number of users, the uniform power allocation at the transmit nodes, $P_{1}=P / N$, is not suitable, and power allocation must be carried out.

Besides, by relaxing the transmit power constraint $\left(P_{1}>\right.$ $P / N)$ the problem of rate loss when the number of source nodes is large can be mitigated, even if the transmit power is uniformly distributed among the source nodes. Looking at the results of Fig. 5 and TABLE III, it is possible to assert that the proposed scheme presents a good tradeoff between transmission rate and BER reliability, even for a large number of users as long as there exists a proper number of antennas at the relay station.

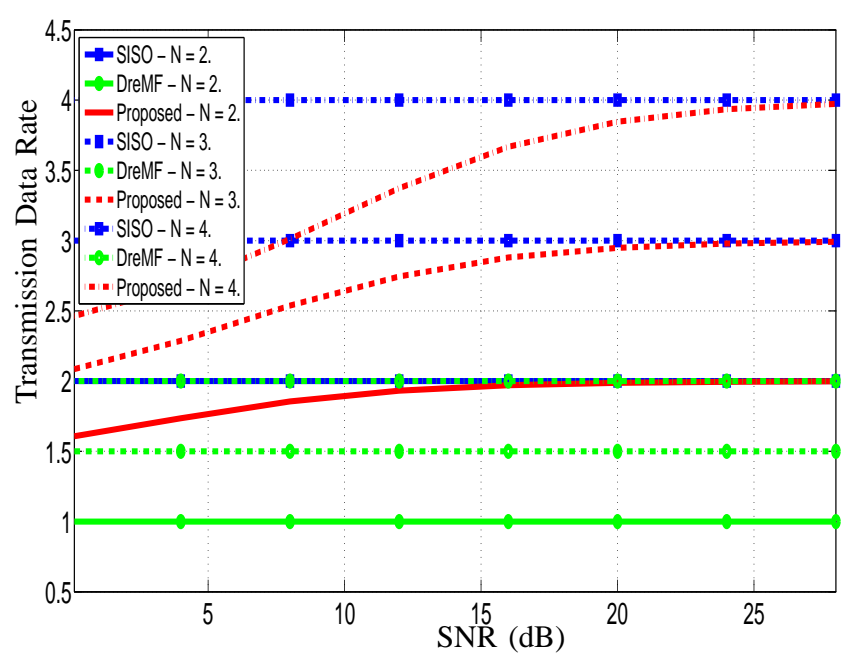

Fig. 5. Transmission rate of the proposed scheme for different number of sources.

TABLE II. RELAY USAGE FOR DIFFERENT NUMBER OF SOURCES.

\begin{tabular}{c|c|c|c|c|c}
\hline \multirow{2}{*}{ Sources } & \multirow{2}{*}{ Relay usage } & \multicolumn{4}{|c}{ Average SNR } \\
\cline { 3 - 6 } & & $0 d B$ & $8 d B$ & $16 d B$ & $24 d B$ \\
\hline \multirow{2}{*}{$\mathrm{N}=2$} & (DreMF) & $60.4 \%$ & $85.5 \%$ & $97.0 \%$ & $99.5 \%$ \\
\cline { 2 - 6 } & (SISO) & $39.6 \%$ & $14.5 \%$ & $3.0 \%$ & $0.5 \%$ \\
\hline \multirow{2}{*}{$\mathrm{N}=3$} & (DreMF) & $38.6 \%$ & $69.1 \%$ & $92.0 \%$ & $98.5 \%$ \\
\cline { 2 - 6 } & (SISO) & $61.4 \%$ & $30.9 \%$ & $8.0 \%$ & $1.5 \%$ \\
\hline \multirow{2}{*}{$\mathrm{N}=4$} & (DreMF) & $22.8 \%$ & $51.5 \%$ & $83.3 \%$ & $96.7 \%$ \\
\cline { 2 - 6 } & (SISO) & $77.2 \%$ & $48.5 \%$ & $16.7 \%$ & $3.3 \%$ \\
\hline
\end{tabular}

TABLE III. TRANSMISSION RATE LOSS FOR AVERAGE SNR $=20 \mathrm{~dB}$.

\begin{tabular}{c|c}
\hline Number of Sources & Rate Loss \\
\hline$N=2$ & 0.012 \\
\hline$N=3$ & 0.051 \\
\hline$N=4$ & 0.144 \\
\hline
\end{tabular}

\section{CONCLUding Remarks}

A cooperative scheme for the time-division multiple-access relay channel with antenna selection at the relay station has been proposed. The scheme can be considered a good solution for networks with a small-to-moderate number of users and with demand for spectral efficiency. It was observed that the proposed scheme one outperforms the SISO and DreMF schemes, and it provides a good tradeoff between spectral efficiency and diversity gain even if there is a large number of users or a few number of antennas deployed at the relay station. It is also worth mentioning that the reliability of the proposed system could be optimized if the fixed transmit power constraint is relaxed. The analytical solution for the proposed scheme is an interesting issue which is already under investigation, as well as the assumption of a real error detector at the destination node for a better system evaluation.

\section{ACKNOWLEDGMENT}

This work was partially supported by the Coordination for the Improvement of Higher Education Personnel (CAPES/PROEX), Brazil, under grant 1345 647, the National Council for Scientific and Technological Developmen (CNPq), Brazil, under grants 211271-2013-6 and 306145/2013-8, the Swedish-Brazilian Research and Innovation Centre (CISB) and Saab AB. 


\section{REFERENCES}

[1] K. J. R. Liu, A. K. Sadek, W. Su, and A. Kwasinski, Cooperative Communications and Networking, 1st ed. Cambridge University Press, 2009.

[2] E. Hossain, D. I. Kim, and V. K. Bhargava, Cooperative Cellular Wireless Networks, 1st ed. Cambridge University Press, 2011.

[3] A. Sendonaris, E. Erkip, and B. Aazhang, "User cooperation diversity part I: System description," IEEE Transaction on Communications, vol. 51, no. 11, pp. 1927-1938, 2003.

[4] J. N. Laneman and G. W. Wornell, "Distributed space-time coded protocols for exploiting cooperative diversity in wireless networks," IEEE Transaction Information Theory, vol. 49, no. 10, pp. 2415-2425, 2003.

[5] Z. Shen, A. Papasakellariou, D. Gerstenberger, and F. Xu, "Overview of 3gpp lte-advanced carrier aggregation for $4 \mathrm{~g}$ wireless communications," IEEE Communications Magazine, vol. 50, no. 2, pp. 122-130, 2012.

[6] L. Xie and P. R. Kumar, "Multisource, multidestination, multirelay wireless networks," IEEE Transactions on Information Theory, vol. 53, no. 10, pp. 3586-3595, 2007.

[7] A. Bletsas, H. Shin, and M. Z. Win, "Cooperative communications with outage-optimal opportunistic relaying," IEEE Transactions on Wireless Communications, vol. 6, no. 9, pp. 3450-3460, 2007.

[8] X. Zhang, W. Wang, and X. Ji, "Multiuser diversity in multiuser two-hop cooperative relay wireless networks: System model and performance analysis," IEEE Transactions on Veihicular Technology, vol. 58, no. 2, pp. 1031-1036, 2009.

[9] A. Vanelli-Coralli, G. E. Corazza, G. K. Karagiannidis, and P. T. Mathiopoulos, "Satellite communications: Research trends and open issues," in Satellite and Space Communications, 2007. IWSSC '07. International Workshop on, Sept. 2007, pp. 71-75.

[10] S. S. Ikki and M. H. Ahmed, "Performance analysis of cooperative diversity with incremental-best-relay technique over rayleigh fading channels," IEEE Transaction on Communications, vol. 59, no. 8, pp. 2152-2161, 2011

[11] A. Bletsas, A. Khisti, D. P. Reed, and A. Lippman, "A simple cooperative diversity method based on network path selection," IEEE Journal on Selected Areas in Communications, vol. 24, no. 3, pp. 659672, 2006.

[12] A. H. Bastami and A. Olfat, "Selection relaying schemes for cooperative wireless networks with adaptive modulation," IEEE Journal on Selected Areas in Communications, vol. 60, no. 4, pp. 1539-1558, 2011.

[13] M. Iezzi, M. D. Renzo, and F. Graziosi, "Network code design from unequal error protection coding: Channel-aware receiver design and diversity analysis," in Communications (ICC), 2011 IEEE International Conference on, June 2011, pp. 1-6.

[14] M. F. Al-Mistarihi, R. Mohaisen, A. Sharaqa, M. M. Shurman, and K. A. Darabkh, "Performance evaluation of multiuser diversity in multiuser two-hop cooperative multi-relay wireless networks using maximal ratio combining over rayleigh fading channels," International Journal of Communication Systems, 2013.

[15] Y. Meng, G. Liu, G. Shen, Z. Liu, Q. Jiang, and Y. Tang, "Superposition coding and analysis for cooperative multiple access relay system," Wireless Personal Communications, vol. 70, no. 2, pp. 1011-1028, 2013.

[16] J. Harshan and B. S. Rajan, "On two-user gaussian multiple access channels with finite input constellations," Information Theory, IEEE Transactions on, vol. 57, no. 3, pp. 1299-1327, March 2011.

[17] A. Winkelbauer and G. Matz, "Joint network-channel coding in the multiple-access relay channel: Beyond two sources," in Communications Control and Signal Processing (ISCCSP), 2012 5th International Symposium on, May 2012, pp. 1-5.

[18] N. Tishby, F. Pereira, and W. Bialek, "The information bottleneck method," in 37th Allerton Conf. on Communication and Computation, Sep 1999, pp. 368-377. 